\title{
Nonlinear Predictive Control of Autonomous Soaring UAVs Using 3DOF Models
}

\author{
Yuyi Liu, Stefano Longo and Eric C. Kerrigan
}

\begin{abstract}
We design a nonlinear model predictive control (NMPC) system for a soaring UAV in order to harvest the energy from the atmospheric updrafts. Our control framework combines an online estimation with a heuristic search method to obtain the UAV optimal trajectory. To allow for real-time computation of the control commands we solve the optimal control problem using a 3 degrees-of-freedom (DOF) model but apply the inputs to a more realistic 6DOF model. Hence, we design a 3DOF-6DOF model interaction strategy. Simulations show how the control system succeeds in energy extraction in a challenging dynamic atmospheric environment while satisfying its real-time contraints.
\end{abstract}

\section{INTRODUCTION}

The endurance of Unmanned Aerial Vehicles (UAVs) is being pursued as a current area of keen interest. A diverse set of mission purposes, such as rescue searching, border patrol, and environmental sensing require UAVs to improve their time aloft [1]. The restrictions of flight time are altitude, fuel and batteries if the human constraints in determining loiter time are ignored. Therefore, it is highly beneficial to improve UAV endurance by executing online estimation of updrafts and harvesting the most amount of energy via optimal control methods [2]. This energy, coming from rising masses of air, is largely unexploited and can be utilised by UAVs to gain altitude, reduce fuel payload and even recharge batteries.

The majority of previous research that focuses on aircraft control employs either a rigid 3DOF or a $6 \mathrm{DOF}$ model. The 3DOF model is faster for computation but less accurate [3], while the 6DOF is more accurate but has only been validated by simple environmental simulations [4]. A 3DOF model is feasible for a real-time control because of its low computational requirements, but the performance of the controller implemented on a UAV has not yet been fully investigated. Compared to the computation cost of a 3DOF model, the cost of a 6DOF model is over 10 times higher [5], although this can be improved using methods like Multiplexed MPC [6].

Previously, research on UAV energy extraction from thermals (rising warm air) has been done via optimal control methods, often with strong assumptions on the thermal model [7], [8]. NASA has conducted a study on the estimation of such thermal energy generated from uneven surface

Y. Liu is with the Department of Aeronautics, Imperial College London, London, SW7 2AZ, UK

yuyi.liulleimperial.ac.uk

S. Longo is with the Department of Automotive Engineering, Cranfield University, Cranfield, MK43 OAL, UK

s.longodcranfield.ac.uk

E. C. Kerrigan is with the Department of Electrical and Electronic Engineering and with the Department of Aeronautics, Imperial College London, London, SW7 2AZ, UK

e.kerrigandimperial.ac.uk heating for the control of a soaring UAV [9]. The latest study regarding the control of energy harvesting method for UAVs has employed an online estimation to simulate the UAV optimal trajectory without assuming a predefined updraft model [2]. However, the updraft models in the majority of previous work are static with no change throughout the duration of the UAV encounters.

In this paper, Nonlinear MPC (NMPC) is used to compute the optimal path for a UAV to extract the maximum amount of energy (potential and kinetic) from updrafts, combined with an online estimation of such updrafts. A heuristic search is employed when certain conditions are fulfilled in order to increase the possibility of the UAV circling around a strong updraft when it attempts to detect the surrounding atmosphere data. For the purpose of computational cost reduction of MPC, an interaction strategy between the 3DOF modelbased controller and the 6DOF plant is proposed. By using a smaller model for the controller, real-time implementation is theoretically possible with limited computational resources. Thus, we solve the optimization problem using a 3DOF (fast computation) but, via a suitable transformation, we apply the control action to a more realistic 6DOF model.

\section{NONLINEAR MODEL PREDICTIVE CONTROL SETUP}

\section{A. Optimal Control Setup}

The in-house Imperial College London Optimal Control Software (ICLOCS) [10] is used to solve the optimal control problem in conjunction with MATLAB. The equation below is employed as the framework upon which the parameters, i.e. the UAV equations of motion, associated constraints and the atmospheric updraft model, are imported. The optimal control problem, in general terms, can be stated as

$$
\begin{aligned}
& \min _{u(t)} J\left(x(\cdot), u(\cdot), t_{0}, t_{f}\right) \\
& \text { s.t.: } \dot{x}=f(x(t), u(t)), \quad \forall t \in\left[t_{0}, t_{f}\right] \\
& \quad g_{L} \leq g(x(t), u(t)) \leq g_{U}, \quad \forall t \in\left[t_{0}, t_{f}\right] \\
& \quad \phi_{L} \leq \phi\left(x_{f}\right) \leq \phi_{U} \\
& \quad x_{L} \leq x(t) \leq x_{U}, \quad \forall t \in\left[t_{0}, t_{f}\right] \\
& \quad u_{L} \leq u(t) \leq u_{U}, \quad \forall t \in\left[t_{0}, t_{f}\right] \\
& \quad u_{0}=u\left(t_{0}\right), \quad x_{0}=x\left(t_{0}\right), \quad x_{f}=x\left(t_{f}\right) .
\end{aligned}
$$

Here, the cost function is set as

$$
J\left(x(\cdot), u(\cdot), t_{0}, t_{f}\right) \triangleq \int_{t_{0}}^{t_{f}} L(x(t), u(t)) d t+E\left(x_{f}\right)
$$


TABLE I

UAV (DG-100 GLIDER) MODEL PARAMETERS.

\begin{tabular}{ll}
\hline Parameter (unit) & Value \\
\hline Mass, $m(\mathrm{~kg})$ & 300 \\
Wing area, $S\left(\mathrm{~m}^{2}\right)$ & 11 \\
Air density, $\rho\left(\mathrm{kg} \cdot \mathrm{m}^{-3}\right)$ & 1.225 \\
Gravitational acceleration, $g\left(\mathrm{~m} \cdot \mathrm{s}^{-2}\right)$ & 9.81 \\
\hline
\end{tabular}

where $E(\cdot)$ is the terminal cost and $L(\cdot)$ is the stage cost function. The cost function is minimized over the timevarying control inputs $u(\cdot)$, and the initial state $x_{0}$. Meanwhile, $g(\cdot)$ describes the general path constraints and $\phi(\cdot)$ imposes the ending boundary conditions for the phase.

\section{B. Unpowered Aircraft Model}

Considering the quest for real-time control, a rigid 3DOF UAV model is employed for the optimal control problem instead of a more computationally intensive 6DOF model, which considers both translational and rotational motions. The 3DOF point mass unpowered flight model consists of 6 nonlinear ordinary differential equations (ODEs) in the inertial coordinate, with certain rate constraints included to ensure that its performance is realistic. Its scalar representation under vertical atmospheric influence (updrafts) [11] is given by

$$
\begin{aligned}
\dot{x} & =V \cos \gamma \cos \chi \\
\dot{y} & =V \cos \gamma \sin \chi \\
\dot{h} & =V \sin \gamma+W_{h} \\
\dot{\gamma} & =\frac{1}{m V}(L \cos \mu-m g \cos \gamma) \\
\dot{\chi} & =\frac{1}{m V \cos \gamma}(L \sin \mu) \\
\dot{V} & =\frac{1}{m}(-D-m g \sin \gamma)
\end{aligned}
$$

where $L=\frac{1}{2} C_{L} \rho S V^{2}$ is the lift and $D=\frac{1}{2} C_{D} \rho S V^{2}$ is the drag of the UAV. The coefficient of lift is $C_{L}=0.7$. $2 \pi \alpha$ where $\alpha$ is the aircraft Angle Of Attack (AOA) and the factor of 0.7 is used to discount for the three-dimensional flow effects on a finite-span wing. The coefficient of drag is $C_{D}=0.01+0.02 C_{L}^{2} . W_{h}$ is the vertical atmospheric effect of the updraft that needs to be estimated. Other parameters are given in Table I corresponding to those of a DG-100 glider. In this 3DOF model, the control input vector is defined consisting of the AOA rate and roll rate as

$$
\boldsymbol{u}_{3 D O F} \triangleq(\dot{\alpha}, \dot{\mu}) .
$$

The state vector is defined as the six states in the equations of motion, three inertial positions, flight path angle, heading angle and the UAV airspeed, together with the AOA and roll angle:

$$
\boldsymbol{x}_{3 D O F} \triangleq(x, y, h, \gamma, \chi, V, \alpha, \mu) .
$$

TABLE II

STATE AND INPUT CONSTRAINTS.

\begin{tabular}{lll}
\hline States (unit) & Lower bound & Upper bound \\
\hline$x(\mathrm{~km})$ & -2 & 2 \\
$y(\mathrm{~km})$ & -2 & 2 \\
$h(\mathrm{~km})$ & 0 & $\infty$ \\
$\gamma\left({ }^{\circ}\right)$ & -30 & 30 \\
$\chi\left(^{\circ}\right)$ & $-\infty$ & $+\infty$ \\
$v(\mathrm{~m} / \mathrm{s})$ & 15 & 70 \\
$\alpha\left(^{\circ}\right)$ & 0 & 10 \\
$\mu\left(^{\circ}\right)$ & -45 & 45 \\
\hline Inputs (unit) & Lower bound & Upper bound \\
\hline$\dot{\alpha}\left({ }^{\circ} / \mathrm{s}\right)$ & -10 & 10 \\
$\dot{\mu}\left({ }^{\circ} / \mathrm{s}\right)$ & -30 & 30 \\
\hline
\end{tabular}

\section{Constraints and cost function}

Table II provides the constraints of the aircraft states employed in the optimal controller, where the bounds of $\pm \infty$ describe the unconstrained states. According to these constraints, the UAV is restricted within a 4-by-4 kilometer square for flight. Considering that the UAV has to fly above a certain height to avoid being in a precarious position, a terminal state bound for the altitude is imposed as

$$
h_{t_{f}} \in[100, \infty) .
$$

The rate constraints are also imposed on input vectors as given in Table II, in order to ensure that the UAV stays within the envelope of a real flight. The cost function is chosen to have only a boundary cost, hence the optimal controller is designed to maximize the amount of atmospheric energy extracted at the end of the horizon, i.e.

$$
\begin{aligned}
& L \triangleq 0, \forall t \in\left[t_{0}, t_{f}\right], \\
& E \triangleq-h_{e}\left(t_{f}\right)=-\left(h_{t_{f}}+\frac{V_{t_{f}}^{2}}{2 g}\right),
\end{aligned}
$$

where $h_{e}$ is the weight-specific energy height, which represents the summation of flight potential and kinetic energy over its weight [12], with the minus sign ensuring the problem can be defined as a minimization problem.

\section{Atmospheric model}

The updraft scenario shown in Figure 1 is a modified MATLAB's peaks function. The model employed demonstrates a difficult problem of multiple updraft cores with different magnitudes and radii, which have overlapping regions of influence and differs from previous research [4]. Meanwhile, downdrafts are inserted and the average magnitude of the distribution is set to be negative. In addition, to simulate the disturbance given by a lateral cross-wind the updraft scenario is set within a continuous lateral-direction motion at $-2.5 \mathrm{~m} / \mathrm{s}$. The influence of wind is added to demonstrate the inherent robustness of the NMPC scheme. 

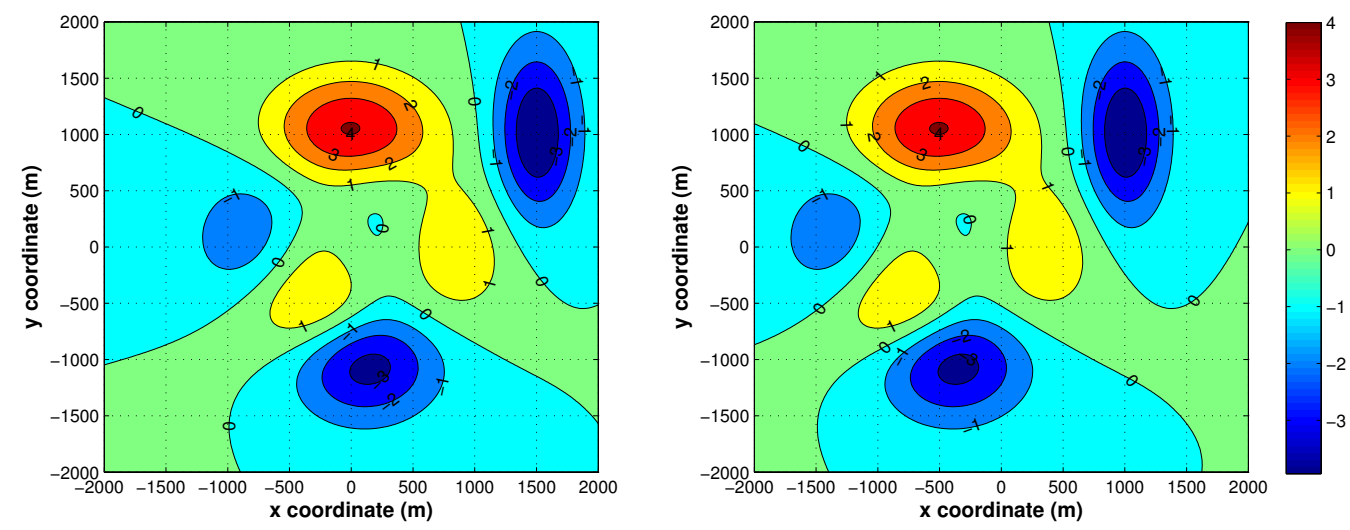

Fig. 1. (Left) Updraft magnitude contour at $\mathrm{t}=0 \mathrm{~s}$. (Right) Updraft magnitude contour at $\mathrm{t}=200 \mathrm{~s}$. The air mass constantly moves at $-2.5 \mathrm{~m} / \mathrm{s}$ in $x$-direction. The units of the contour ( $h$ direction) are $\mathrm{m} / \mathrm{s}$.

\section{E. Online Estimation and Heuristic Search Model}

An approach to estimate the updraft distribution is required for the UAV when not given the full environment information through any external support. Therefore, a twolayer Generalised Regression Neural Network (GRNN) is introduced to regress the velocity data of the updrafts that the UAV traverses and thus generate a 'picture' of the surrounding updraft distribution for the controller to solve the optimization problem.

In this simulation the UAV is restricted to the updraft velocity data measured via sensors along the flight path only, which is different from other studies in which aircrafts are assumed to be equipped with special tools such as remotesensing infrared thermal cameras [4].

To execute the optimal search with an online estimation scheme that satisfies the time-critical nature of the UAV controller, a heuristic search technique is used to ensure that:

1) the UAV can fly a trajectory to obtain sufficient information for the online estimation model so that the globally optimal region can be found;

2) the UAV can find a sufficiently strong updraft for energy extraction.

The heuristic search is determined by the following:

1) only if the UAV is at or higher than a 'safe altitude', it starts to consider the search of updrafts;

2) the heuristic search is executed if the Root Mean Squared Error (RMSE) between the measured and estimated updraft is larger than an 'RMSE threshold', or if the maximum measured updraft velocity is less than 'a reasonable expected updraft velocity';

3) a reference direction is generated for the UAV to fly for the heuristic search time.

As listed in Table III, the heuristic search parameters are selected to make the UAV soar around an updraft core when the estimate of the updrafts scenario is accurate enough and that the updraft found is strong enough for the UAV to extract a reasonable amount of energy.
TABLE III

HEURISTIC SEARCH MODEL PARAMETERS.

\begin{tabular}{ll}
\hline Parameter (unit) & Value \\
\hline Safe altitude $(\mathrm{m})$ & 500 \\
RMSE threshold $(\mathrm{m} / \mathrm{s})$ & 1 \\
Reasonable updraft strength $(\mathrm{m} / \mathrm{s})$ & 2.5 \\
Heuristic search time $(\mathrm{s})$ & 60 \\
\hline
\end{tabular}

\section{3DOF AND 6DOF MODEL INTERACTION STRATEGY}

To satisfy the real-time requirements, a 3DOF model is used for the optimal control problem. The calculated UAV's inputs are applied to a more realistic 6DOF model. Hence, an interaction strategy has to be designed in order to match the difference of states and inputs between the two models, as described next.

\section{A. 6DOF Unpowered UAV Model}

The 6DOF aircraft model is a set of equations of motion with 12 states and 3 input vectors. To obtain the transformation from the $3 \mathrm{DOF}$ problem directly, the $6 \mathrm{DOF}$ model is defined in the inertial frame as:

$$
\begin{aligned}
& \dot{x}=V \cos \gamma \cos \chi \\
& \dot{y}=V \cos \gamma \sin \chi \\
& \dot{h}=V \sin \gamma+W_{h} \\
& \dot{\gamma}=\frac{1}{m V}(L \cos \mu-m g \cos \gamma \\
& -Y \sin \mu \cos \beta) \\
& \dot{\chi}=\frac{1}{m V \cos \gamma}(L \sin \mu+Y \cos \mu \cos \beta) \\
& \dot{V}=\frac{1}{m}(-D-m g \sin \gamma+Y \sin \beta) \\
& \dot{\alpha}=q-(p \cos \alpha+r \sin \alpha) \tan \beta \\
& +\frac{1}{m V \cos \beta}(-L+m g \cos \gamma \cos \mu)
\end{aligned}
$$




$$
\begin{aligned}
& \dot{\mu}=(p \cos \alpha+r \sin \alpha) \sec \beta \\
& +\frac{1}{m V}[-m g \cos \gamma \cos \mu \tan \beta+L(\tan \gamma \sin \mu+\tan \beta) \\
& +Y \tan \gamma \cos \mu \cos \beta] \\
& \dot{\beta}=p \sin \alpha-r \cos \alpha \\
& +\frac{1}{m V}(m g \cos \gamma \sin \mu+Y \cos \beta)
\end{aligned}
$$

where $Y=\frac{1}{2} C_{Y} \rho S V^{2}$ is the side force of the aircraft and $C_{Y}=-\frac{1}{8} \pi \beta$, the sideslip coefficient, which is a function of the sideslip angle $\beta$. All other symbols are defined the same as in a $3 \mathrm{DOF}$ model. The control input vector is the time derivatives of three angular velocity components, considering that the parameters of the UAV, such as aerodynamic moment and aircraft inertia matrix components, are not given:

$$
\boldsymbol{u}_{6 D O F} \triangleq(\dot{p}, \dot{q}, \dot{r}) \text {. }
$$

The states are

$$
\boldsymbol{x}_{6 D O F} \triangleq(x, y, h, \gamma, \chi, V, \alpha, \mu, \beta, p, q, r) .
$$

where $x, y, h$ are three inertial positions, $\gamma, \chi, V, \alpha, \mu, \beta$ are the flight path angle, heading angle, the UAV airspeed, AOA, roll angle and sideslip angle, respectively, The first 8 states are same as the states of the 3DOF model, hence they can be used without transformation. Variables $p, q, r$ are the rotational moment components of the UAV.

\section{B. Overview of Model Interaction Strategy}

Assuming there is no sideslip for the unpowered UAV because of its symmetric structure, $\beta, \dot{\beta}$ and the sideslip force are fixed to zero while

$$
\begin{aligned}
\dot{\alpha} & =q+\frac{1}{m V}(-L+m g \cos \gamma \cos \mu) \\
\dot{\mu} & =(p \cos \alpha+r \sin \alpha)+\frac{1}{m V}(L \tan \gamma \sin \mu) \\
\dot{\beta} & =p \sin \alpha-r \cos \alpha+\frac{g}{V} \cos \gamma \sin \mu \equiv 0
\end{aligned}
$$

The angular velocity components $p, q$ and $r$ can be transformed from the control input $\dot{\alpha}$ and $\dot{\mu}$ as

$$
\begin{aligned}
& p=\dot{\mu} \cos \alpha-\frac{g}{V} \cos \gamma \sin \mu \sin \alpha \\
& -\frac{1}{m V}(L \tan \gamma \sin \mu \cos \alpha) \\
& q=\dot{\alpha}-\frac{1}{m V}(-L+m g \cos \gamma \cos \mu) \\
& r=\dot{\mu} \sin \alpha+\frac{g}{V} \cos \gamma \sin \mu \cos \alpha \\
& -\frac{1}{m V}(L \tan \gamma \sin \mu \cos \alpha) .
\end{aligned}
$$

Then, the inputs $\dot{p}, \dot{q}$ and $\dot{r}$, which are used in the plant model, can be obtained via the forward finite difference equation

$$
\dot{f} \cong \frac{f_{i+1}-f_{i}}{\delta t},
$$

where $\delta t$ is the interval between two control commands.

Hence, we built a strategy to calculate the control input in a 3DOF-model-based controller and to simulate a real UAV

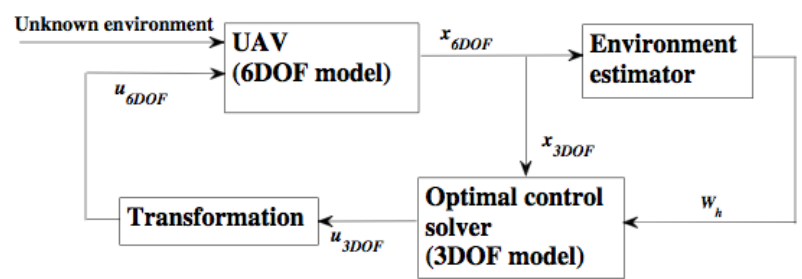

Fig. 2. NMPC structure of the $3 \mathrm{DOF}$ and $6 \mathrm{DOF}$ model interaction strategy.

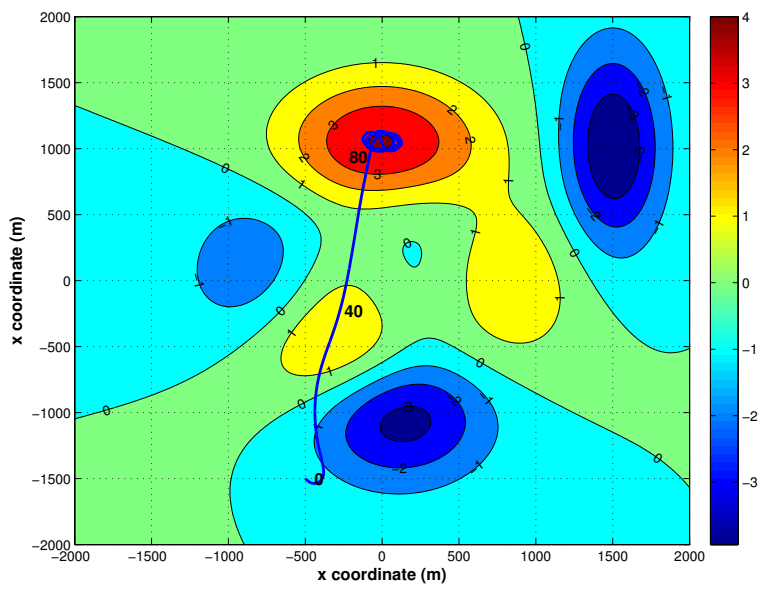

Fig. 3. Optimal trajectory of the UAV using a 6DOF model succeeds in converging to the strongest updraft when given full knowledge. The units of the contour ( $h$ direction) are $\mathrm{m} / \mathrm{s}$.

with a $6 \mathrm{DOF}$ model. This 3DOF and 6DOF model interaction strategy (shown diagrammatically in Figure 2) could allow the applicability of a NMPC algorithm on a computationally inexpensive real-time controller for a UAV.

\section{ApPlicAtion OF NMPC TO THE UNPOWERED AUTONOMOUS UAV}

\section{A. Simulation with Full Updraft Knowledge}

The performance of the interaction strategy is tested via the simulation of the UAV, first with full updraft distribution information, in order to check the interaction between the 6DOF UAV model and the 3DOF-model-based optimal controller. No online estimation or heuristic search method is employed at this stage. The UAV is released close to the strongest downdraft in the environment. The first simulation uses a 3DOF model (same as the model in the controller), while the second simulation employs the model interaction strategy and thus the 6DOF model for the actual motions. The closed-loop NMPC results are obtained from implementing the optimal controller in a receding horizon fashion with a sampling time of 2 seconds. The prediction horizon of the controller is $120 \mathrm{~s}$. It is observed from Figure 3 that the UAV succeeds in tracking and finally converging to the strongest updraft. The energy harvesting performance for both models (3DOF and 6DOF) is similar (a comparison is shown in Figure 4). For the first $20 \mathrm{~s}$, the UAV performs the same avoidance maneuver and it traverses the intermediate updraft 


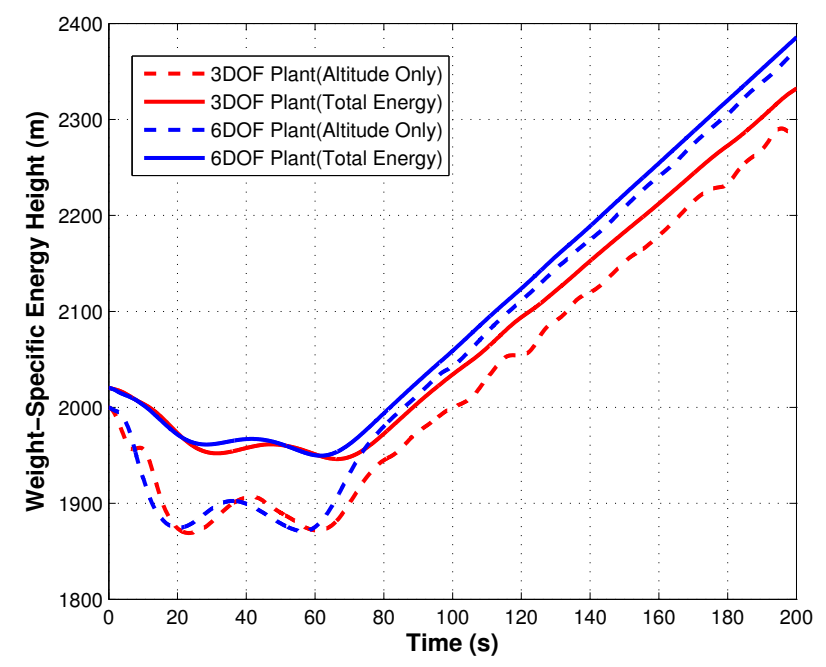

Fig. 4. Comparison of the performance of MPC structures using 3DOF and $6 \mathrm{DOF}$ models in simulation with full updraft knowledge. The units of the contour ( $h$ direction) are $\mathrm{m} / \mathrm{s}$.

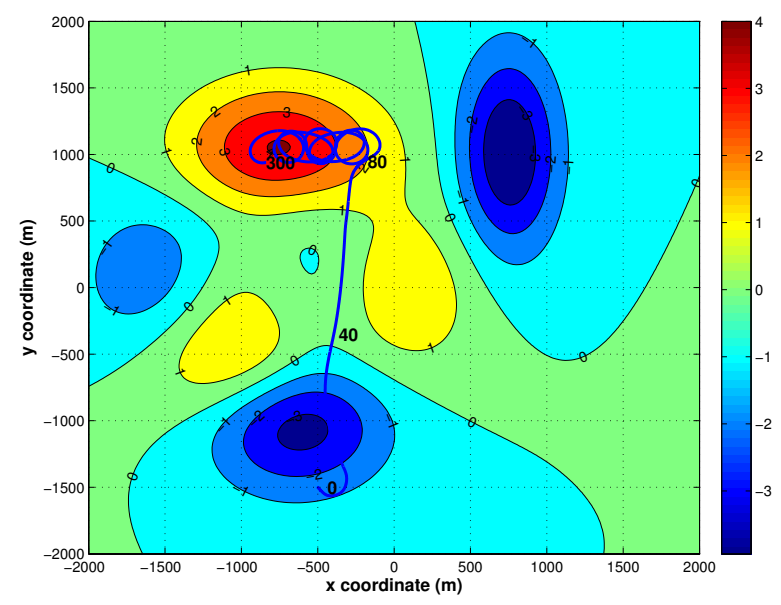

Fig. 5. Optimal trajectory of the UAV after $300 \mathrm{~s}$ in a constant moving updraft environment. The air mass constantly moves at $-2.5 \mathrm{~m} / \mathrm{s}$ in the $x$ direction. The units of the contour ( $h$ direction) are $\mathrm{m} / \mathrm{s}$.

core to increase the altitude and thus its potential energy. The UAV finally circles around the strongest updraft core and gains energy from it until the end of the simulation. The difference between the two sets of simulations caused by the input transformation is acceptable considering that the UAV is still able to reach its goal.

\section{B. Simulation in a Moving Environment with Full Updraft Knowledge}

In the second set of simulations, a moving updraft scenario is employed in order to assess the UAV's performance under a more challenging but realistic environment. The air mass constantly moves at $-2.5 \mathrm{~m} / \mathrm{s}$ in the $x$-direction. As seen in Figure 5, the UAV first reaches the strongest updraft at around $80 \mathrm{~s}$, then it follows the horizontal motion of the

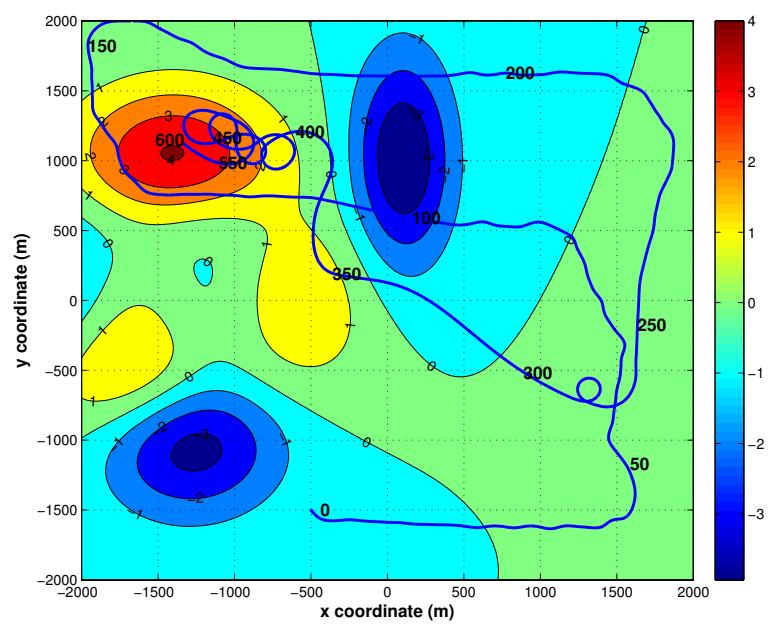

Fig. 6. UAV trajectory at $600 \mathrm{~s}$ overlaid upon a constant moving updraft environment. The air mass constantly moves at $-2.5 \mathrm{~m} / \mathrm{s}$ in the $x$-direction. The units of the contour ( $h$ direction) are $\mathrm{m} / \mathrm{s}$.

updraft scenario and succeeds in tracking it to obtain the maximum potential energy.

\section{Simulation of Heuristic Search with Online Estimation in Moving Environment}

Previous research [2] described the feasibility of the heuristic search model with online estimation in a static atmospheric scenario. The updraft information is estimated via the GRNN and the heuristic search activates once the conditions are met. The updraft scenario is in constant motion as in the previous section. The systematic search model with an adaptive grid method has a satisfying performance in the moving scenario. The online estimation method succeeds in updating the "picture" when the strongest updraft core is moving, avoiding the UAV soaring around the region that is first detected as the destination, but which has already moved away.

In the simulations, the environment is first divided into 600-by-600 meter grid squares. In order to increase the possibility of finding the strongest updraft, while avoiding the downdrafts, an adaptive grid method is introduced by coarsening the grid space to 800 -by- 800 meter when the UAV discovered that the updraft velocity reduces or does not increase much over the past 20 seconds (updraft gradient is low or negative). On the contrary, the estimator would refine the grid to 400 -by-400 meter size elements when the thermal gradient is strong and positive in the past 20 seconds. Thereafter, the UAV has the ability to negotiate the low-speed updrafts and downdrafts faster, while searching the strong updraft region in detail.

The UAV's trajectory at the end of $600 \mathrm{~s}$ can be observed in Figure 6. After a 400 s' search, the UAV succeeded in reaching the optimal point and started soaring around the updraft core. However, when it updates the updraft picture estimated at the beginning of the next heuristic search (after $60 \mathrm{~s}$ ), the strongest updraft had already moved away, hence 


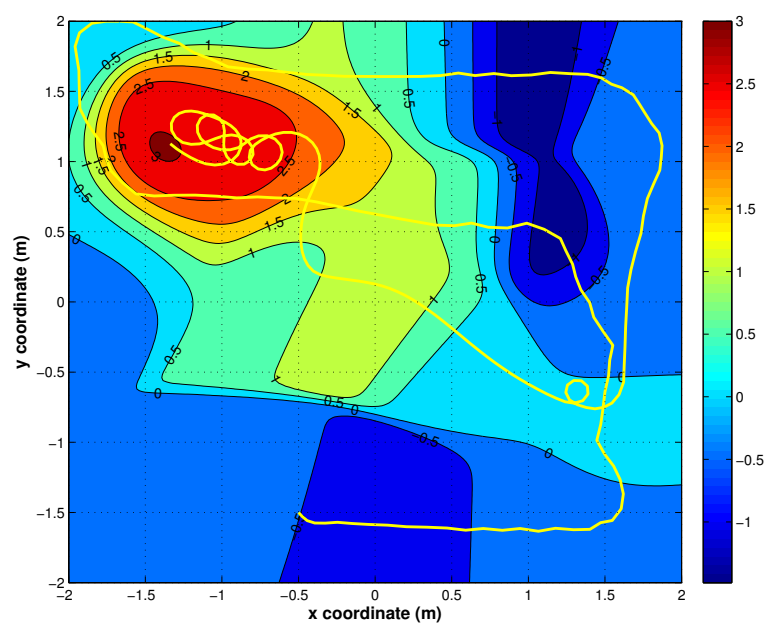

Fig. 7. UAV trajectory at 600 s overlaid upon the estimated updraft environment. The units of the contour ( $h$ direction) are $\mathrm{m} / \mathrm{s}$.

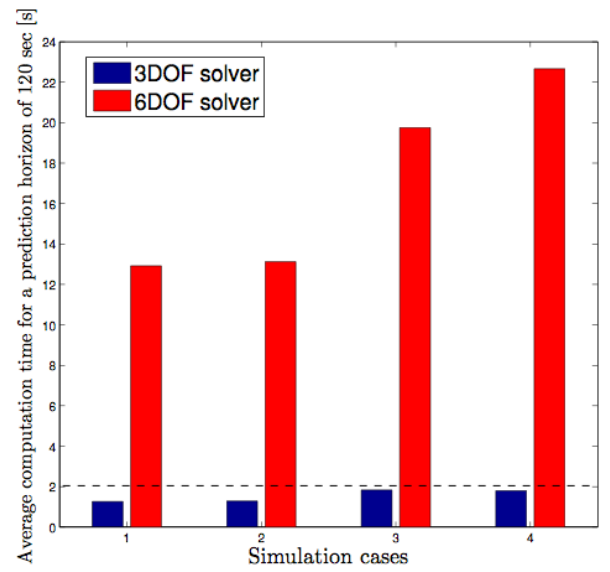

Fig. 8. Comparison of computation times: 3DOF solver vs 6DOF solver. Case1: with full knowledge in static air mass. Case2: with full knowledge in moving air mass. Case3: without full knowledge in static air mass. Case4: without full knowledge in moving air mass.

the UAV started to chase it in order to extract sufficient energy from the environment. Analysing the estimated updraft distribution, the estimation (Figure 7) is quite close to the real environment, particularly the moving centre of the strongest updraft core. This suggests that the UAV succeeds in following the environmental motion without any special sensing equipment.

\section{Performance of $3 D O F$ and $6 D O F$ Model Interaction Strategy}

A pivotal factor of a real-time controller is its computational time. The application of a 3DOF model with the interaction strategy allows the computation of the optimal solution to be 10 times faster than the situation where a 6DOF model is used. We compare the average computation time for a solution with a 3DOF and 6DOF model in Figure 8 . Since the selected sampling time is $2 \mathrm{~s}$, it is easy to see that this could have not been achieved using a $6 \mathrm{DOF}$ model. Our interaction strategy dramatically speeds up the solver. The less than 2 seconds average computation time of the 3DOF solver suggests that it is theoretically feasible to use this optimal energy-harvesting strategy for real-time UAV control.

\section{CONCLUSIONS}

We have shown that the application of nonlinear and constrained optimal control techniques on UAVs to extract the energy from the atmospheric updrafts can be a promising method to enhance flight endurance, loiter time and to reduce fuel consumption. In addition, the control approach under limited resources has shown to have the potential of being implemented in real-time via a 3DOF-6DOF model interaction strategy. The application of an adaptive search grid for the environmental exploration on the UAV has shown to give a good performance in a challenging and realistic dynamic atmospheric scenario. Future work could include an implementation of the proposed method into embedded hardware.

\section{ACKNOWLEDGMENTS}

This research has been supported by the EPSRC grant number EP/G031576/1, EP/F041004/1 and the European Union Seventh Framework Programme FP7/2007-2013 'EMBOCON' under grant agreement number FP7-ICT-2009-4 248940 .

\section{REFERENCES}

[1] N. Slegers, J. Kyle, and M. Costello, "Nonlinear model predictive control technique for unmanned air vehicles," Journal of Guidance, Control, and Dynamics, vol. 29, no. 5, pp. 1179-1188, 2006.

[2] D. Lee, S. Longo, and E. C. Kerrigan, "Predictive control for soaring of unpowered autonomous UAVs," in Proc. $4^{\text {th }}$ IFAC Nonlinear Model Predictive Control Conference, Netherlands, 2012.

[3] N. Akhtar, J. F. Whidborne, and A. K. Cooke, "Real-time optimal techniques for unmanned air vehicles fuel saving," Proc. IMechE Journal of Aerospace Engineering, vol. 226, no. 10, pp. 1315-1328, 2011

[4] N. Akhtar, A. K. Cooke, and J. F. Whidborne, "Positioning algorithm for autonomous thermal soaring," AIAA Journal of Aircraft, vol. 49, no. 2, pp. 472-482, 2012.

[5] Y. Liu, "Control of autonomous soaring gliders," Master's thesis, Imperial College London, UK, 2012.

[6] H. Richter, A. Singaraju, and J. S. Litt, "Multiplexed predictive control of a large commercial turbofan engine," Journal of Guidance, Control, and Dynamics, vol. 31, no. 2, pp. 273-281, 2008.

[7] Y. C. Qi and Y. J. Zhao, "Energy-efficient trajectories of unmanned aerial vehicles flying through thermals," Journal of Aerospace Engineering, vol. 18, no. 2, pp. 84-92, 2005.

[8] N. E. Kahveci, P. A. Ioannou, and M. D. Mirmirani, "Adaptive LQ control with anti-windup augmentation to optimize UAV performance in autonomous soaring applications," IEEE Trans. Control Systems Technology, vol. 16, no. 4, pp. 691-707, 2008.

[9] M. J. Allen and V. Lin, "Guidance and control of an autonomous soaring UAV,” NASA, TM-2007 - 214611, Tech. Rep., 2007.

[10] P. Falugi, E. C. Kerrigan, and E. V. Wyk. (2010) Imperial College London Optimal Control Software (ICLOCS). [Online]. Available: http://www.ee.ic.ac.uk/ICLOCS/

[11] R. B. Patel, "Prohibited volume avoidance for aircraft," Ph.D. dissertation, Imperial College London, UK, 2010.

[12] Y. J. Zhao, "Optimal patterns of glider dynamic soaring," Optimal Control Applications and Methods, vol. 25, pp. 67-89, 2004. 
2013-07-22

Nonlinear predictive control of autonomous soaring UAVs using 3DOF models

Liu, Yuyi

Institute of Electrical and Electronics Engineers

Liu, Y., Longo, S., Kerrigan, E. C. (2013) Nonlinear predictive control of autonomous soaring UAVs using 3DOF models, European Control Conference 2013 (ECC'13), 16-19 July 2013, Zurich, Switzerland

https://dspace.lib.cranfield.ac.uk/handle/1826/10883

Downloaded from Cranfield Library Services E-Repository 